\title{
Article \\ pH-Dependent Formation of Oriented Zinc Oxide Nanostructures in the Presence of Tannic Acid
}

\author{
Nurul Akmal Che Lah ${ }^{1, * \mathbb{D}}$, Aqilah Kamaruzaman ${ }^{1}$ and Sonia Trigueros ${ }^{2,+} \mathbb{D}$ \\ 1 Faculty of Manufacturing \& Mechatronics Engineering Technology, Universiti Malaysia Pahang, Pekan, \\ Pahang 26600, Malaysia; aqilah996@gmail.com \\ 2 Department of Zoology, University of Oxford, Oxford OX1 3PS, UK; sonia.trigueros@zoo.ox.ac.uk \\ * Correspondence: akmalcl@ump.edu.my; Tel.: +60-9424-5825 \\ + Current address: Structural Biology Department, Molecular Biology Institute of Barcelona (IBMB), \\ Spanish National Research Council (CSIC), 08028 Barcelona, Spain.
}

Citation: Che Lah, N.A.; Kamaruzaman, A.; Trigueros, S. pH-Dependent Formation of Oriented Zinc Oxide Nanostructures in the Presence of Tannic Acid. Nanomaterials 2021, 11, 34. https://dx.doi.org/10.3390/ nano11010034

\section{Received: 28 October 2020}

Accepted: 24 November 2020

Published: 25 December 2020

Publisher's Note: MDPI stays neutral with regard to jurisdictional claims in published maps and institutional affiliations.

Copyright: () 2020 by the authors. Licensee MDPI, Basel, Switzerland. This article is an open access article distributed under the terms and conditions of the Creative Commons Attribution (CC BY) license (https:/ / creativecommons.org/ licenses/by/4.0/).

\begin{abstract}
To crucially comprehend the relaying factors behind the growth mechanism of $\mathrm{ZnO}$ nanostructures, the needs to understand the cause of preferences in the enhancement of desired physicochemical properties are essential. The particular oriented attachment (OA) is believed to become the cause of the classical growth pattern of $\mathrm{ZnO}$ nanostructures which is mainly controlled by the Ostwald ripening (OR) process. In the present work, the concerns over the systematic changes in size and the morphological surface of $\mathrm{ZnO}$ nanostructures upon exposure to tannic acid (TA) prepared by drop-wise method turns the particles to different surface adjustment state. Here, we assessed the TA capping ability and its tendency to influence the OA process of the $\mathrm{ZnO}$ nanostructures. The detailed process of the growth-based TA system via transmission electron microscopy (TEM), scanning electron microscopy (SEM), and FFT autocorrelation revealed the $\mathrm{pH}$ effect on their physical properties which proved the transition surface properties state of the particles from rough to smooth states due to oriented attachment. For pure $\mathrm{ZnO}$ nanostructures, the surface is almost smooth owing to the strong bonding particles which are then changed to coarsened surface structures upon the introduction of TA. Strong surface adsorption of $\mathrm{Zn}$ cations and phenol ligands mediated the agglomerated nanocrystals, surprisingly with smaller nanostructures dimension.
\end{abstract}

Keywords: $\mathrm{ZnO}$ nanostructures; growth mechanism; tannic acid; oriented attachment; Ostwald ripening

\section{Introduction}

To date, the oriented attachment (OA) or oriented agglomeration growth mechanism developed by Penn and Banfield et al. [1-3] has been widely employed to demonstrate the specific assembly of the quantum system into aggregated nanocrystals including the $\mathrm{ZnO}$ nanostructures semiconductor. The assembly involves the first-order phase transition from an individual seed crystal of $\mathrm{ZnO}$ nucleated upon the exposure of heat that proceeded to the construction of further defect surfaces and the crystal morphology through coalescing, recrystallize, or assemble into larger structures. The occurrence of defect surfaces due to the created edge dislocations that are always observed in most of the secondary nanocrystals (metastable structures) yields the possibility of incorporating the defects into initially defect-free nanoparticles due to the extended lattices through the preferential attachment on specific crystal faces. Generally, this OA crystal growth mechanism is distinct from but fundamentally controls by the classical Ostwald ripening (OR) that provides a route by which distinctive crystal morphologies and nano-architectures can be produced [4-6]. These colloidal $\mathrm{ZnO}$ nanosheets growth in solution is the result of the combined fundamental effect of OR and the unique OA processes in which the dissolution, precipitation, and ripening during the particle motion, collision, and agglomeration processes formed those unique crystallographic orientations. Hence, the difference is that the formation of 
complex nanostructure crystals with wide varieties morphologies including rods, chains, multipods, and branched nanowires are mainly explained by OA, which cannot be justified by classical OR [7-9].

$\mathrm{ZnO}$ is one of the vital materials in semiconductor therefore becomes one of the leading contenders in micro-electric industries materials. The formation of functional surface $\mathrm{ZnO}$ nanostructures through $\mathrm{OA}$ to construct hierarchically structured $\mathrm{ZnO}$ in aid of surfactant has been rapidly growing $[10,11]$. The unique properties of the $\mathrm{ZnO}$ semiconductor are caused by the generation of novel phenomena driven by interactions at their interfaces due to the presence of surfactants in between the aligned nanostructures, that promote the OA based on surfactant interaction-related driving forces for self-assembly formation, kinetic stabilization between the intermediate nanoparticles, and control of coalescence preferential crystal face or the morphology of the nanocrystal $[9,12,13]$. The hexagonal wurtzite-type structure of $\mathrm{ZnO}$ has the $C_{6 v}^{2}(P 63 m c)$ space group, with two formula units in the unit cell with all atoms occupying $C_{6 v}^{2}$ composed of alternating planes of tetrahedral coordinated by $\mathrm{O}^{2-}$ and $\mathrm{Zn}^{2+}$ ions in an $\mathrm{ABAB}$ pattern (hexagonal close packing) at $\mathrm{c}-$ axis $[14,15]$. The produced dipole moment normal to the basal (0001) plane is common for $\mathrm{ZnO}$ nanostructures induced by the opposite stack charged ions. This type of nanoassembly could yield better unique materials which give broader application prospects based on their optical, magnetic, electrical, and chemical properties.

The physical OA arrangement of $\mathrm{ZnO}$ can be observed by several microscopy techniques. Herein, the main focus of this study is the effect of optimized $\mathrm{pH}$ conditions on suspension and dispersion of $\mathrm{ZnO}$ nanostructures upon the addition of TA. The dynamic changes in the relationship between particle structure and the agglomeration growth were dictated by analyzing the OA process of crystal growth formation (individually and aggregate clusters). This study focuses on a better understanding of $\mathrm{ZnO}$ nanostructures growth from physio-chemical dispersion stability and colloidal suspension morphologies. The results described in this study track the sequence of crystal growth provided as clear evidence that $\mathrm{OA}$ rarely operates as the sole crystal growth mechanism and open better understanding of $\mathrm{ZnO}$ crystal growth by $\mathrm{OA}$.

\section{Materials and Methods}

\subsection{Materials}

ZnO-tannic acid (TA) nanostructures were prepared using the sol-gel method with additional sodium citrate (SC) acting as surface modification agent in an aqueous solution. All chemicals used were of analytical grade and without further purification. Bulk $\mathrm{ZnO}$ $\left(d_{m} \approx 50 \mu \mathrm{m},>99 \%\right.$, Merck, UK) is used as a precursor and $\mathrm{SC}\left(\mathrm{C}_{6} \mathrm{H}_{5} \mathrm{Na}_{3} \mathrm{O}_{7} \cdot 2 \mathrm{H}_{2} \mathrm{O},>99 \%\right.$, R\&M Chemical, Semenyih, Malaysia) as a stabilizer. TA $\left(\mathrm{C}_{76} \mathrm{H}_{52} \mathrm{O}_{46}\right)$ powder (R\&M Chemical, Semenyih, Malaysia) is used as surface modification with distilled water (Material Laboratory Faculty of Manufacturing and Mechatronics Engineering Technology, Universiti Malaysia Pahang) is used as a solvent.

\subsection{Preparation of $\mathrm{ZnO}-\mathrm{TA}$ Nanostructures}

About $4 \mathrm{mM}$ bulk $\mathrm{ZnO}(50 \mu \mathrm{m})$ is vigorously mixed and added drop-wise with $2 \mathrm{mM}$ SC along with slow stirring for $20 \mathrm{~min}$ at $50{ }^{\circ} \mathrm{C}$ to obtain a homogenous mixture. When the white suspension is formed we continuously stirred it for a period of $6 \mathrm{~h}$. Stock standard solutions of each chemical reactant are produced straightly by weighing and dilution with $\sim 90 \%$ water to a known volume. The white color sediment formed is centrifuged $(12,000 \mathrm{rpm}$ for $12 \mathrm{~min})$ and rinsed a few times with distilled water. The aim is to wash off those impurities that caused improper low stability. The reaction continued for different reaction temperatures $\left(60,70,80\right.$, and $\left.90^{\circ} \mathrm{C}\right)$ sequentially. To study the effect of TA solution on the synthesized $\mathrm{ZnO}$ nanostructures, each concentrated sample is diluted with $50 \mathrm{~mL}$ of distilled water, TA was added drop-wise to check its influence on agglomeration formation of $\mathrm{ZnO}$ nanostructures. The as-synthesized $\mathrm{ZnO}$ nanostructures sample are monitored 
in comparison with initial concentration-reactant samples $(2.5 \mathrm{mM})$ produced at various temperatures. The added concentrations of TA are 10, 50, 100, and $200 \mu \mathrm{L}$.

\subsection{Characterisation of ZnO-TA Nanostructures}

The characterizations of pure $\mathrm{ZnO}$ and $\mathrm{ZnO}-\mathrm{TA}$ nanostructures are carried out using the following instruments. Transmission electron microscopy (TEM-2010, JEOL Ltd., Tokyo, Japan): Grids were examined and all micrographs recorded with an SIS Mega view III camera. The spots were obtained on formvar-coated 200 mesh copper grids (TAAB, Berks, UK) and post-stained with distilled water. Zeiss field emission scanning electron microscopy (FESEM): with an operating voltage at $30 \mathrm{kV}$ equipped with EDX (SIGMA/VP, Darmstadt, Germany) EDS was used to characterize samples morphology.

\section{Results}

\subsection{The Precipitation of $\mathrm{ZnO}$ Nanostructures}

The identification of the controlled growth and agglomeration mechanisms of wurtzite $\mathrm{ZnO}$ nanocrystals in the presence of TA has been planned based on the implemented experimental work. The primary attention was dedicated to analyzing the initial stages of agglomeration. The hydrothermal reaction between bulk $\mathrm{ZnO}$ (precursor) and distilled water (reactant medium) can be expressed based on these equations:

$$
\begin{gathered}
\mathrm{ZnO}+\mathrm{H}_{2} \rightarrow \mathrm{Zn}\left((\mathrm{OH})_{2}\right) \\
\mathrm{Zn}\left((\mathrm{OH})_{2}\right)+\mathrm{C}_{6} \mathrm{H}_{5} \mathrm{O}_{7} \mathrm{Na}_{3} \rightarrow \mathrm{C}_{6} \mathrm{H}_{7} \mathrm{O}_{8} \mathrm{Na}_{3}+\mathrm{H}_{2} \mathrm{O}
\end{gathered}
$$

The morphology and particle size distribution of the pure $\mathrm{ZnO}$ and $\mathrm{ZnO}-\mathrm{TA}$ nanostructures where the zero-dimensional structures are visible after a short time of hydrothermal processing is shown in Figure 1. For pure $\mathrm{ZnO}$ nanostructures synthesized at 50, 70, and $90{ }^{\circ} \mathrm{C}$ (Figure $1 \mathrm{~A}-\mathrm{C}$ ), it is proved that the morphological shape of the structures is irregular and polygonal which have polygonal modal mean particle size diameters, $d_{m}$ of about $8 \pm 0.2,8 \pm 0.4$, and $13 \pm 0.5 \mathrm{~nm}$, respectively. During the synthesis of pure $\mathrm{ZnO}$ under short processing times and low target temperatures $\left(50\right.$ and $\left.70^{\circ} \mathrm{C}\right)$, the particles are not fully evolved. For most of the captured spots, particles are individually assembled and undergo much slower rates of agglomeration, despite their proximity. As small as $5 \mathrm{~nm}$ particle diameters have been observed in this study. The formation of this critical size is affected by a range of critical factors including the initial degree of supersaturation, reaction temperature, and $\mathrm{pH}$ of the surrounding medium, which are described by classical nucleation theory. The metastable state between the nucleation stage and OR growth stage in a colloidal system is controlled by the thermodynamical growth of OA [16,17]. Initially, these small $\mathrm{ZnO}$ nanostructures with negatively charged SC $\left(\mathrm{C}_{6} \mathrm{H}_{5} \mathrm{O}_{7}{ }^{3-}\right.$ ions) surface surfactants would repel the particles from each other, thus hindering the OR growth from promoting self-orientation of OA growth exclusively. It is believed that the SC monolayer adsorbed on the surface of $\mathrm{ZnO}$ nanostructure is composed of dangling di-hydrogen anions $\left(\mathrm{H}_{2}\right.$ Citrate-), and their central carboxylate ions set out to functionalize the surface atoms of $\mathrm{Zn}$. As the synthesis reaction temperature is changed to $90^{\circ} \mathrm{C}$, the coalescence events of small particles are observed producing larger $d_{m}$ of $\mathrm{ZnO}$ nanostructures and the particles are more firmly attached. This attachment, reminiscent of twinning, occur at their strong assembly appearance yielding in compacting the bundles of attached polygonal nanostructures. It is noted that the particles undergo a process known as crystal facet matching that creates a twin interface of crystal facets via a transient jump to contact behavior which established the OA process. The particles spontaneously attached to form nano polygonal chains in an oriented direction despite the presence of SC ligands. As the crystal facet produced the twin crystal facet interface, the created connective neck between the particles disappears through a rapid diffusion of surface atoms, yielding the formation of a more massive individual particle. 

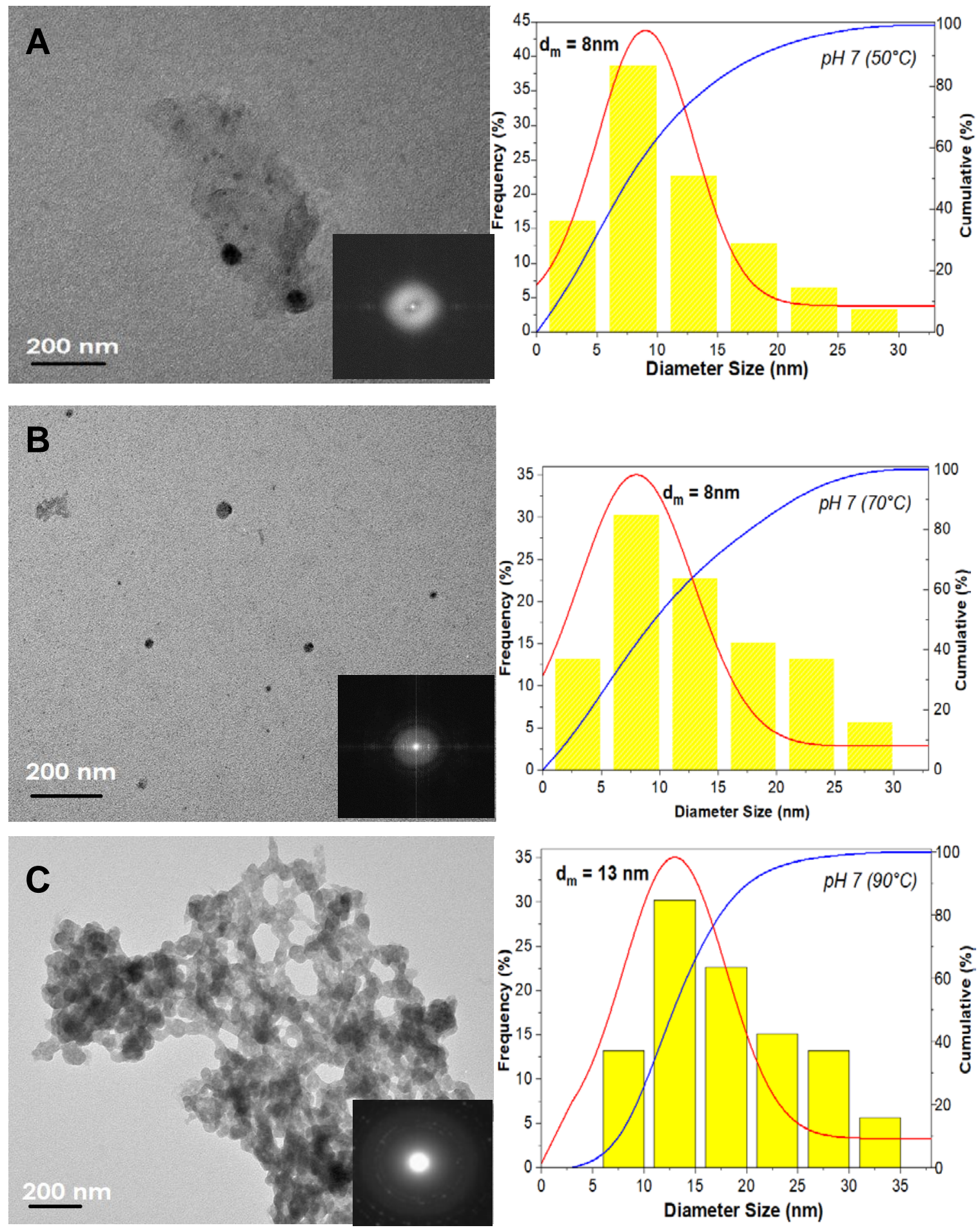

Figure 1. Cont. 

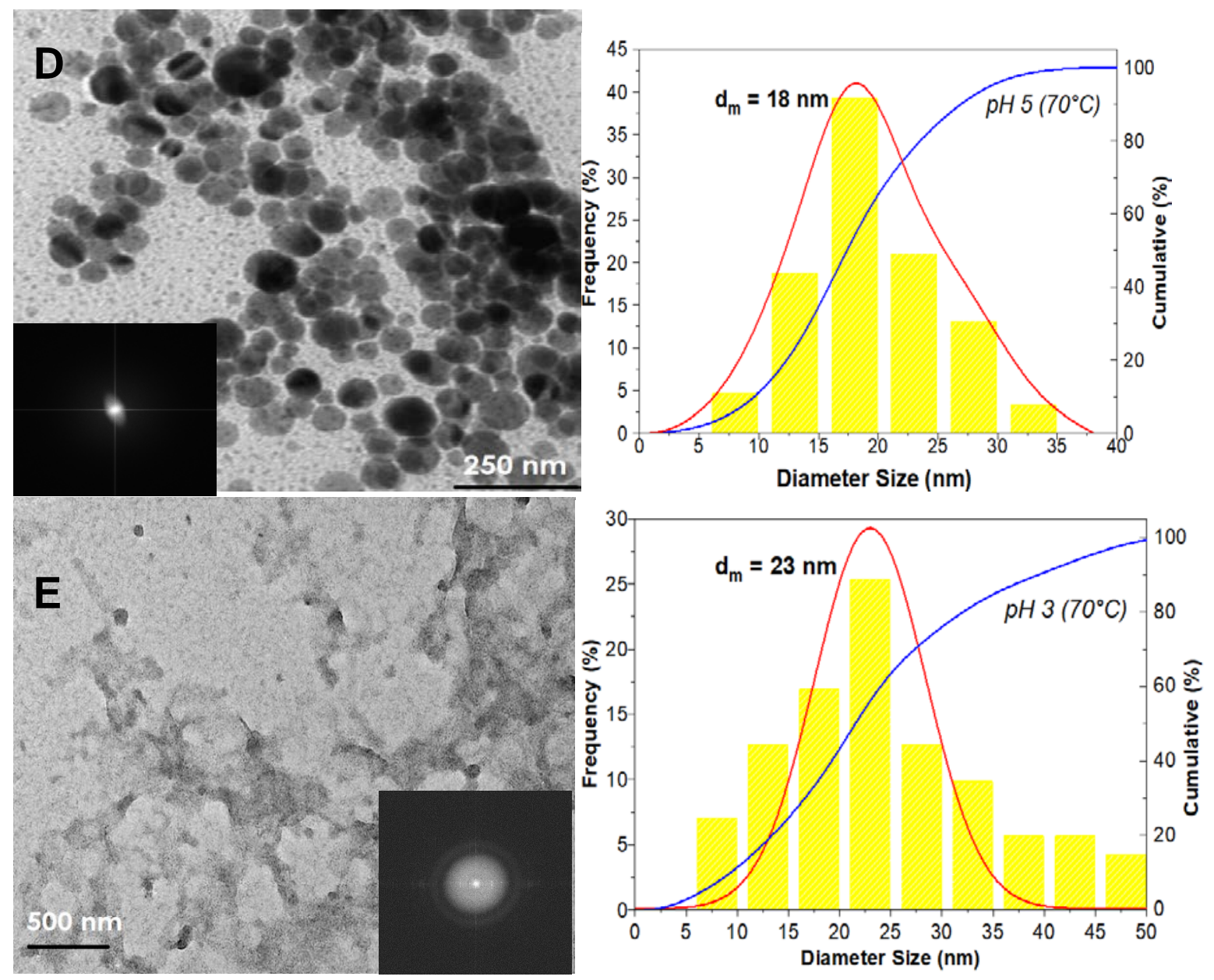

Figure 1. TEM images of pure $\mathrm{ZnO}$ and $\mathrm{ZnO}-\mathrm{TA}$ nanostructures showing particle size distribution plots based on the Gaussian distribution factor (red line) with cumulative analysis (blue line). Pure ZnO nanostructures are synthesized at (A) 50, (B) 70, and (C) $90^{\circ} \mathrm{C}$. Meanwhile, the representative sample of $\mathrm{ZnO}$-TA nanostructures is made using pure $\mathrm{ZnO}$ nanostructures synthesized at $70{ }^{\circ} \mathrm{C}$ which yield $\mathrm{pH}$ value of (D) 5 and (E) 3 . The inset shows the SAED pattern for the respective $\mathrm{ZnO}$ samples.

In the present study, SC shows a vital role in adjusting the morphology and the size of $\mathrm{ZnO}$ samples. The well-known process for the morphological and size changes have been reported in several publications $[13,18,19]$. A similar process is adopted in the present study, but for $\mathrm{ZnO}$ particles not $\mathrm{Zn}$ ion, to produce the $\mathrm{ZnO}$ nanostructures which is affected by the SC. In the presence of SC, the citrate anions adsorb metal surface of $\mathrm{Zn}$ in the absence of $\mathrm{Zn}$ ion thus forming a relatively stable metal-citrate complex in an acidic condition that activates the generation of a smaller unit of bulk $\mathrm{ZnO}$ because of the reaction of $\mathrm{Zn}\left((\mathrm{OH})_{2}\right)$ precipitate and $\mathrm{C}_{6} \mathrm{H}_{5} \mathrm{O}_{7}{ }^{3-}$ ions. It caused the further dissolution of $\mathrm{Zn}(\mathrm{OH})_{2}$, and the formation of small $\mathrm{ZnO}$ occurred at the same time. The $\mathrm{C}_{6} \mathrm{H}_{5} \mathrm{O}_{7}{ }^{3-}$ ions preferentially adsorb on the zinc (001) basal plane as a capping agent [18] which then suppresses the crystal growth along of the (001) preferential direction to form $\mathrm{ZnO}$ nanostructures. Within a short period of reaction time ( $30 \mathrm{~min}$ in our study), these nanostructures aggregate and self-assembled. In my opinion it would be better is you say aggregate or self-assembled, even better just self-assembled into metastable polygonal through the OA for minimizing the total energy of the system.

However, TEM images show that prepared $\mathrm{ZnO}$ nanostructures retained the polygonal clusters that are more aggregated for $\mathrm{ZnO}-\mathrm{TA}$ nanostructures made at $\mathrm{pH} 5$ (Figure 1D) and $\mathrm{pH} 3$ (Figure 1E) with the $d_{m}$ of about $18 \pm 1.5$ and $23 \pm 1.5 \mathrm{~nm}$, respectively. In our opinion the formation of a high surface-free energy created by the ultra-small size distribution 
$\left(d_{m} \sim 8 \mathrm{~nm}\right)$ of $\mathrm{ZnO}$ nanostructures expedited the coalescence of adjacent nanostructures. Furthermore, the presence of TA that creates high, medium acidity (low $\mathrm{OH}^{-} / \mathrm{H}^{+}$ratio) in between the atomic $\mathrm{ZnO}$ nanostructures induced the numerous coordinative unsaturated sites on the $\mathrm{ZnO}$ surface facilitating particle coalescence at specific facets to create prealignment. This condition implies the consequence of surface ligands introduced in the system during $\mathrm{OA}$, regardless of the ligand types, they bond to the surface of $\mathrm{ZnO}$. Hence, desorption of ligands are appraised as to their surfaces that come into contact. The strong force binding would be one of the fundamental factors for the creation of $\mathrm{ZnO}$ nano chains via the OA-based growth mechanism. The selected area electron diffraction (SAED) pattern proves the availability of hexagonal wurtzite crystallites structures owned by $\mathrm{ZnO}$ based on the characteristic diffraction of a ring pattern (inset in Figure 1). The brighter ring and more prominent spots showed the existence of some larger crystallites. The observed rings were relatively continuous, which denoted the presence of small nanocrystallites in a random orientation.

It is revealed that the polycrystalline hexagonal wurtzite structure of $\mathrm{ZnO}$ nanostructures with the observed peak positions in agreement with reported data in Joint Committee on Powder Diffraction Standards (JCPDS, card no: 043-0002 obtained from the library) as indicated in Figure 2. Noted that, for diffractogram test, the amount of sample is expanded for pure $\mathrm{ZnO}$ nanostructures with the total of five pure samples synthesized at temperatures of 90, 80, 70, 60, and $50^{\circ} \mathrm{C}$ as shown in Figure 2A. Three prominent peaks correspond to reflections from (100), (002), and (101) atomic planes of $\mathrm{ZnO}$ phase. It shows the stability, possible directions for grain growth, and are appointed as minimum energy growth phases of $\mathrm{ZnO}$ crystal. The presence of other low-intensity reflections corresponds to (102), (110), (103), (200), (112), and (201) atomic planes of hexagonal ZnO lattice. In all the test samples for pure $\mathrm{ZnO}$ nanostructures, no peak corresponding to other phases or element/impurity emerged in the XRD analysis. Surprisingly, further added TA concentration in as-synthesized $\mathrm{ZnO}$-TA nanostructures samples inhibits the dominancy of the crystalline phase by vanishing the peak intensities. It is observed that the addition of TA does dispel the diffraction peaks positions of the $\mathrm{ZnO}$ nanostructures as demonstrated in Figure 2B,C for samples made at $\mathrm{pH} 5$ and $\mathrm{pH} 3$, respectively, which further implied the absence of any significant lattice on the $\mathrm{ZnO}$ wurtzite structure. Only one sample from $\mathrm{pH} 5$ shows the presence of complete $\mathrm{ZnO}$ atomic planes. It is believed that during the deposition of the samples on the glass substrate, the amount of $\mathrm{ZnO}$ dropped is lower than the TA molecule. Thus, only TA molecules with amorphous spectrum structure in nature are exhibited as they imprinted over $\mathrm{ZnO}$ nanostructures in accordance with FESEM analysis.

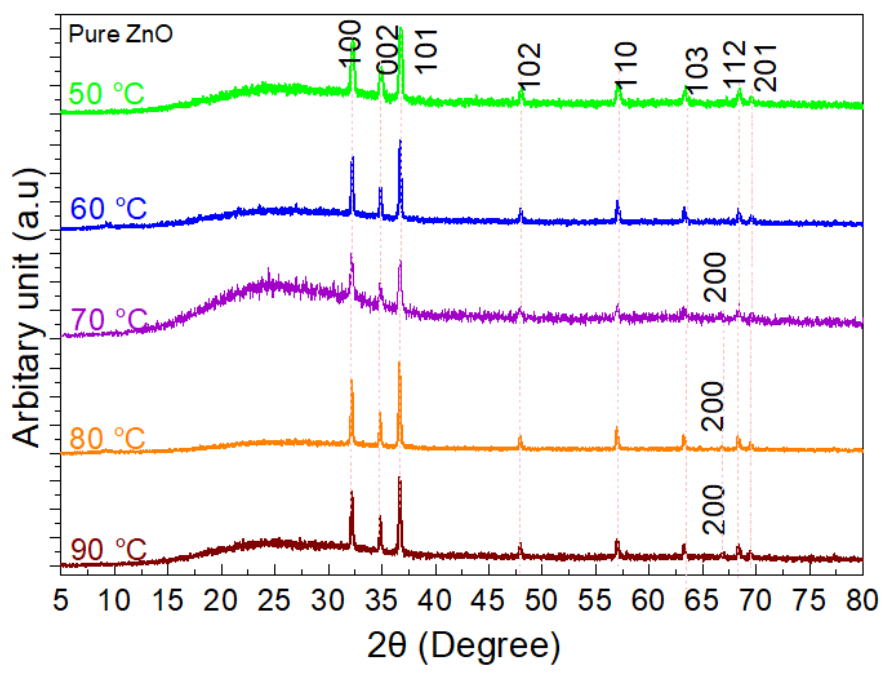

(A)

Figure 2. Cont. 


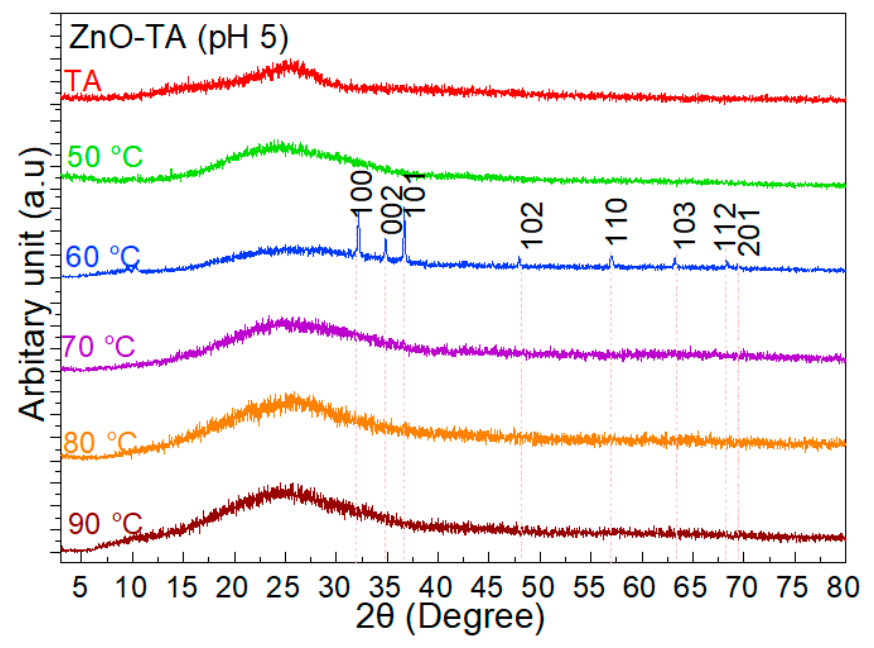

(B)

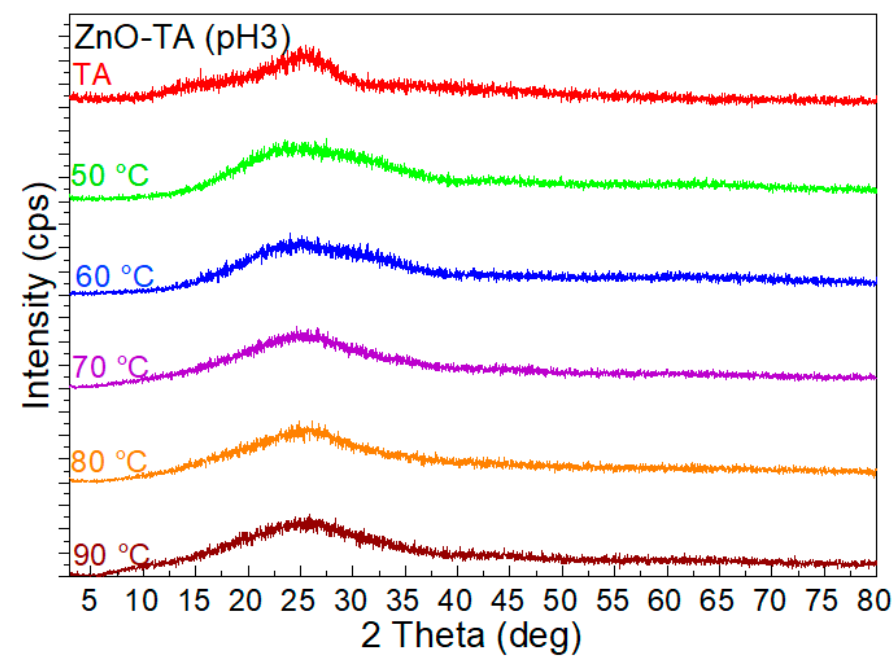

(C)

Figure 2. The $\mathrm{XRD}$ diffractograms for (A) pure $\mathrm{ZnO}$ nanostructures synthesized at 50, 60, 70, 80, and $90{ }^{\circ} \mathrm{C}$ with (B) the XRD peaks of $\mathrm{ZnO}-\mathrm{TA}$ prepared at $\mathrm{pH} 5$ and $(\mathbf{C}) \mathrm{pH} 3$ using pure $\mathrm{ZnO}$ nanostructures synthesized at $50,60,70,80$, and $90^{\circ} \mathrm{C}$. No responses relating to $\mathrm{Zn}$ or $\mathrm{ZnO}$ have been observed in some of the samples prepared at $\mathrm{pH} 5$ and $\mathrm{pH} 3$, suggesting the domination of TA in all samples.

\subsection{Assembly of Pure $\mathrm{ZnO}$ and $\mathrm{ZnO}-\mathrm{TA}$ Nanostructures}

\subsubsection{Influence of the Reaction Temperature}

$\mathrm{ZnO}$ nanostructures obtained at different reactant temperatures exhibited remarkable activity caused by the active surfaces. Under the setup temperature and indirect generated pressure due to the heat, they automatically underwent similar "nanoparticlenanochain-agglomerate-polygon array" structural evolution as represented in Figure 3. First, the crystallization-driven self-assembly of the individual tiny $\mathrm{ZnO}$ nanoparticles changed into $\mathrm{ZnO}$ nano chains. The TEM micrographs demonstrated in Figure 3A-D show the change of $\mathrm{ZnO}$ nanostructures assembly due to the influence of reactant temperatures at $60,70,80$, and $90^{\circ} \mathrm{C}$. The $\mathrm{ZnO}$ nano chains that were created in $\mathrm{H}_{2} \mathrm{O}$, SC, and TA possess irregular curves line, not in straight lines. The $\mathrm{ZnO}$ nano chains consist of many small irregularly crystallized $\mathrm{ZnO}$ nanoparticles with $\mathrm{d} \_\mathrm{m}$ the range below $20 \mathrm{~nm}$ fused together and established a short net-like morphology (Figure 3A). In particular, the $\mathrm{ZnO}$ nano chains formed that contained several straight lines that usually created from the round shape of nanoparticles $\left(d_{m}<10 \mathrm{~nm}\right)$ were connected with each other from different angles. The TEM micrograph in Figure 3B,C showed that the small $\mathrm{ZnO}$ nanoparticles coalesced with each 
other and induced OA process. The typical crystallographic orientation is observed at the boundary among the nanoparticles. This aggregated process exactly represented the common growth mechanism of OA [20]. Similar OA mechanism took place for the $\mathrm{ZnO}$ nanoparticles as the temperature increases (Figure 3D). As noted beforehand, OA growth is the intermediate thermodynamically metastable state between the ripening nucleation process stage and OR growth stage in a dispersed medium nanosystem [16,21,22].

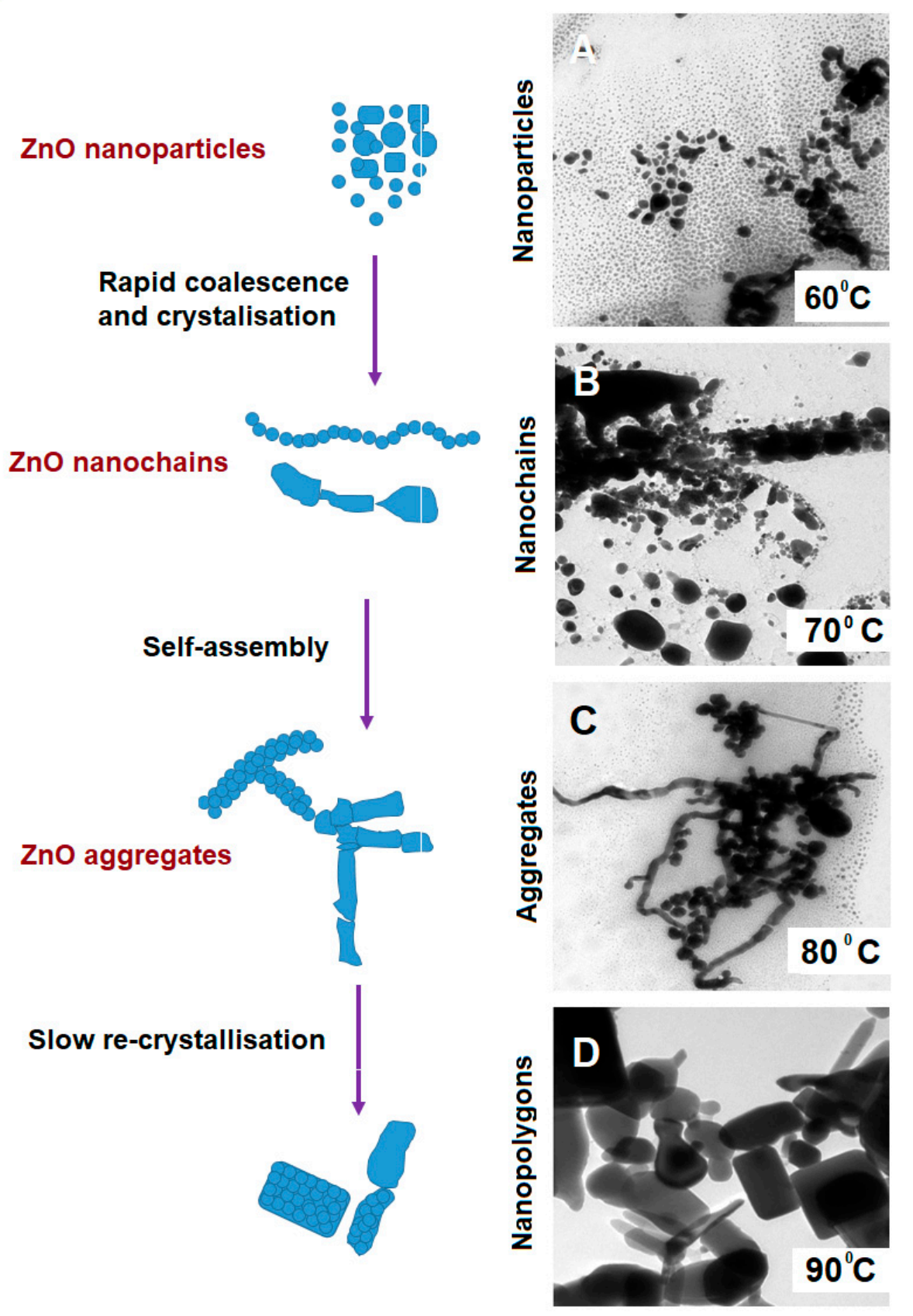

Figure 3. Schematic illustration on the spontaneous growth of $\mathrm{ZnO}$ nanoparticles under the governance of the OA mechanism and the corresponding TEM micrographs on $\mathrm{ZnO}$ microstructures. Noted that the rapid coalescence occurs as the process changes from (A) to (B) in the increase of heat. As the process continues, the self assembly occurs (C) and further caused slow re-crystallisation as the heat is increased (D).

At the initial phase, it is proved that the tiny individual $\mathrm{ZnO}$ nanoparticles possess strongly charged surface surfactants that tend to impede the OR growth (diffusion- 
controlled system growth) which exclusively mediate the OA mechanism. Nonetheless, our results showed that the $\mathrm{ZnO}$ nanoparticles are attracted to each other to construct oriented nano chains structure despite the presence of capping ligands. It is supposed that the small size nanoparticles with a narrow size distribution of $\mathrm{ZnO}$ nanoparticles condense the surface-free energy that activated and accelerated the coalescence between the adjacent nanoparticles. Furthermore, the bonding between the $\mathrm{ZnO}$ nanoparticles and the solvent molecules from the surrounding medium is facilitated by the numerous active surface sites The strong interaction forces created between the passivated surface of uncapped $\mathrm{ZnO}$ nanoparticles and solvent molecules are strong enough to overcome the different density of nanoparticles and to drive the agglomeration process of $\mathrm{ZnO}$ nano chains.

Under static liquid conditions, nanoparticles at close distances tend to agglomerate by moving toward each other, with the facets usually rotating until they achieved their preferred crystal direction alignment, and increased the plan-edge matching for OA determination. Generally, the agglomeration starts with small nanoparticles $\left(d_{m}<10 \mathrm{~nm}\right)$, loosely packed in a pentagonal configuration under slow rate, despite their proximity. It is noteworthy that, at the area of the high local concentration of nanoparticles, the Brownian diffusivity is decreased $[23,24]$. Thus, the OA mechanism is sometimes hindered by these nanoparticles pentagonal configuration, which restricts the orientation of the facet alignment with the neighboring particles. Also, the effect of the physical stirring during the synthesis reaction caused the misalignment. Apart from the existence of electrostatic repulsion surrounding the nanoparticles, van der Waals forces are the primary driving force to rapid agglomeration [25-27].

The stirring rotation mainly influences the agglomeration speed during the reaction. The dissipated heat energy created by the nanoparticles enables them to overcome the electrostatic kinetic barrier to agglomeration. In static conditions, the binding on the surface limits the rotation. Only when the hydrodynamic forces overcome the binding force, nanoparticles tend to rotate and yield rapid orientation of nano alignment easily. This effect gives significant consequence for very close inter-particle distances, at the order of the particle size. This indicates that hydrodynamic forces play an important role in affecting the motion and alignment of nanoparticles during the stirring period. Thus, induced rapid OA aggregative growth with concurrent surface growth, yield from the multimodal particle size distribution [28-30]. The agglomeration may take some time which is estimated to be slower due to the stirring-induced assembly alignment. It is speculated that the primary cause for the OA mechanism is when the combined factors such as the concentration of particles and rapid stirring during mixing, gives significant crystallization pathway. This observation suggested that the OA mechanism occurred in addition to OR, in the presence of slow dissolution and further recrystallisation process.

\subsection{2. $\mathrm{pH}$ Influence}

It has been proposed that the propensity to undergo OA is dependent upon the anisotropy of nanoparticle surface. In this study we show that suspensions with low $\mathrm{pH}$ values (3-5) caused those nanostructures edges to decrease through the slight dissociation of phenolic hydrogen groups in the $\mathrm{pH}$ range of natural water, lowering Coulombic repulsion and yielding the kinetically favored banded agglomeration around the edges [31,32]. This indicated that the introduction of a higher concentration of TA would lead to smaller nanostructures as the higher concentration of TA leads to the rapid formation of a greater number of nuclei.

The adsorbed TA anionic species play a significant role in the OA process mediating the interaction between the acid of the edge phenolic hydrogen group. For instance, phenols are weak acid and lower the $\mathrm{pH}$ of the solution., At pH 5, the TA caused an increased precipitation of $\mathrm{ZnO}$ nanostructures, as shown in Figure 4. When the $\mathrm{pH}$ adjustment was made, the $\mathrm{ZnO}$ nanostructures formed with a light brown color suspension. The color of the suspension varied according to the $\mathrm{pH}$ value: $\mathrm{pH}$ 6, a light brown; $\mathrm{pH}$, a pale dark brown; pH 3, a deep dark brown. The deep dark brown suspension formed that consists 
of $\mathrm{ZnO}$ and TA is a consequence of the action of TA. This action is a result of the effect of the colloidal association of the TA and zinc hydroxide molecules. For pH range of 5 to 3 , the dark brown precipitation formed when the $\mathrm{ZnO}$ was oxidized equitably quickly to zinc hydroxide and the TA was mostly undissociated. It should be noted that only at $\mathrm{pH}$ more than 7 the formation of $\mathrm{ZnO}$ complexes $[33,34]$ takes place as they are highly oxidized and slowly lost from the solution. As the complexes formed with the oxidized $\mathrm{ZnO}$, the $\mathrm{Zn}$ ions are released, and black $\mathrm{ZnO}$ tannate is precipitated. Therefore, at $\mathrm{pH}$ lower than 3 where no complexes were formed, it is believed that abundance of $\mathrm{ZnO}$ had been adjusted to $\mathrm{pH}$ to 6 and 7 as explained elsewhere [35-37] but not included in the present work.
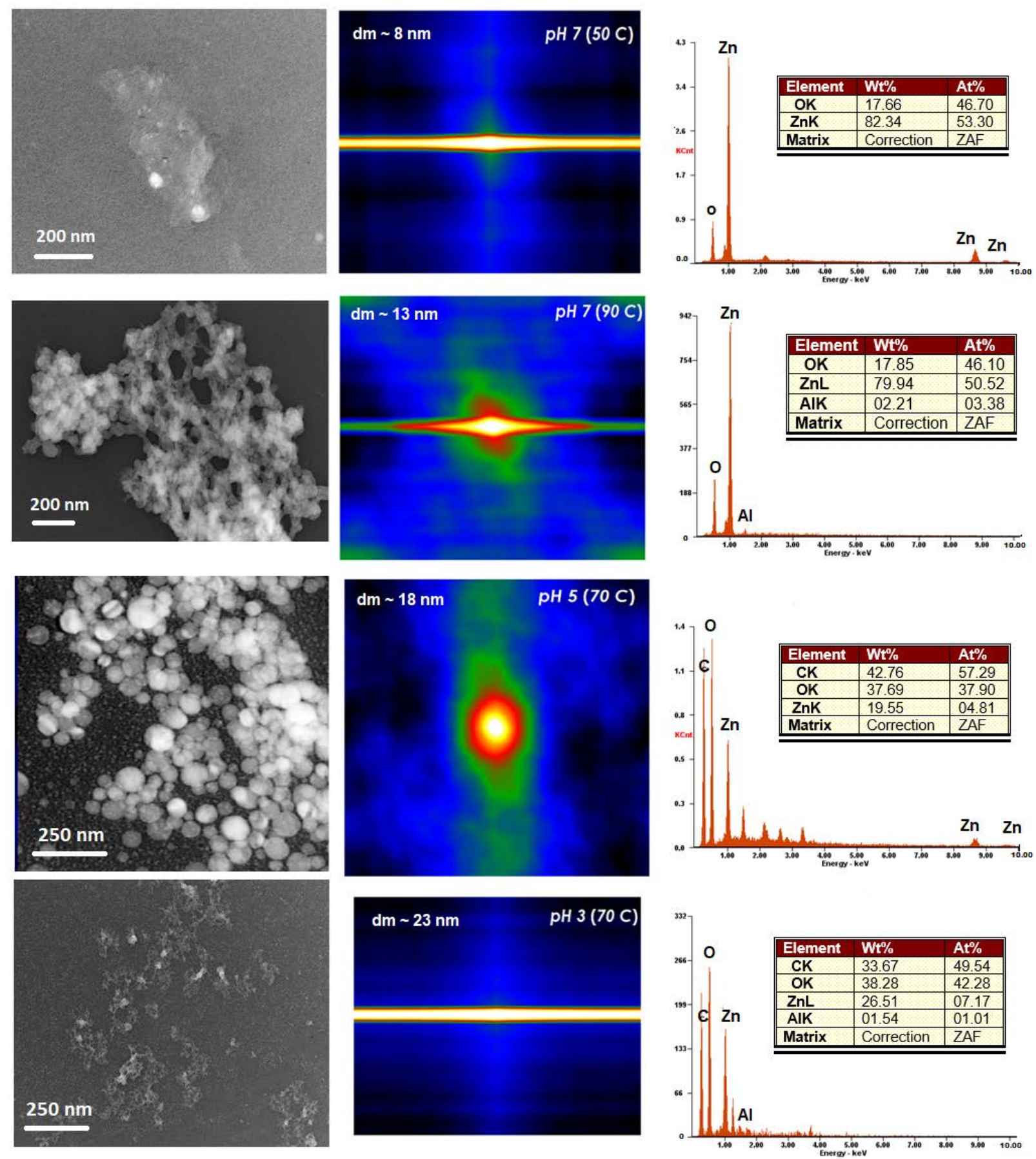

Figure 4. Display of the micrograph TEM images with direct autocorrelation performed on the chosen region. 
Notably, the difference between pure $\mathrm{ZnO}$ and $\mathrm{ZnO}-\mathrm{TA}$ nanostructures is the higher rate of agglomeration in acidic medium. Hence, using polymer ligand that causes the acid interaction may induce $\mathrm{OA}$ and increase the ratio of basal to edge interaction. At $\mathrm{pH} 3$, all the $\mathrm{ZnO}$ was oxidized by the time it contacted with TA. Noted that the rate of oxidation of the $\mathrm{ZnO}$ nanostructures upon the introduction of $\mathrm{TA}$ is a function of $\mathrm{pH}$ and the amount of TA itself. At pH 6 with not sufficient amount of TA, the oxidation rate was slowed and a minor effect was observed because of the reducing power of the TA. As the amount of TA increased, which decreased the $\mathrm{pH}$ number, the rate of the oxidation increased as supported by the EDX reports (right) from SEM images.

Noted that the definition of "agglomeration" and "aggregation" is different based on the International Union of Pure and Applied Chemistry (IUPAC) for nanostructures. Aggregation is defined as the joining of nanostructures through a strong interparticle bonding, causing irreversible clustering. Whereas, agglomeration is where the dispersed particles in the liquid are brought together through weak bond interaction which could also result in a phase separation known for its reversibility. Therefore, it is deduced that lowering the $\mathrm{pH}$ caused a decrease in the weak interactions (e.g., hydrogen bonding) between the nanostructures. Since there is a direct contact between the TA and $\mathrm{ZnO}$ atoms upon the reaction, the TA had a remarkable role in governing the reversible agglomeration and dispersion behavior of the prepared $\mathrm{ZnO}$ nanostructures.

According to the results above, it is concluded that the $\mathrm{pH}$ shows the pure $\mathrm{ZnO}$ nanostructures is dissimilar from that of the $\mathrm{ZnO}-\mathrm{TA}$ nanostructures and that the TA is the leading cause for the new behavior of the ZnO-TA nanostructures. The TA attaches to the surface of $\mathrm{ZnO}$ nanostructures via hydrogen bond interactions due to the abundance of phenolic groups after the exposure of $\mathrm{ZnO}-\mathrm{TA}$ nanostructures.

\subsection{Autocorrelation}

Direct fast Fourier transform (FFT) autocorrelation started with the analysis of the TEM micrograph images, as shown in Figure 4. The direct autocorrelation function from the micrograph images was obtained from the function tab of autocorrelation using Gatan Suite Software. As the autocorrelation determines the self-similarity of an image, the result always shows a peak at the center (each image point is correlated to itself) and an additional structure that ranges from an amorphous background, for a random distribution of dots, to an ordered peak array, for an ordered lattice of dots. The autocorrelation of the micrograph region indicates a definite bright peak of correlation in the middle that is similar to the respective area from the original micrograph. The condition is always observed for autocorrelation functions as, the midpoint where the image is focused and been shifted either in $x$ - or $y$-axis directions.

As observed from the micrographs and autocorrelation images, the sides of the mid contours are believed to represent the preferred distance to the nearest other nanostructural units in the image. Since $\mathrm{ZnO}$ are crystalline materials, a long-range order which defines the crystalline in the peaks is consistent with the reported work. The long contours in the $x$-axis line would correspond to the size of the structures visible in the image region. Nonetheless, the areas around the central contour may then indicate the existence of a second layer of nanostructure which is usually partially visible as proved by the TEM micrographs. The FFT autocorrelation method is a rapid technique with less aliasing problem. However, there is an obvious aliasing problem for samples $\mathrm{pH} 7\left(50^{\circ} \mathrm{C}\right)$ and $\mathrm{pH} 3\left(70^{\circ} \mathrm{C}\right)$ that affects the direct FFT correlation method. The effect is observed by the rise in the $x$-axis as a straight line due to the non-crystalline nature of the oxide caused by the discrete in-built function of the FFT method. The autocorrelation function analysis on the $\mathrm{ZnO}$ surface provides no evidence of dislocations nor discontinuous coverage. These data suggest that the thin surface layer consists of crystalline, not any sort of carbonaceous materials.

Noted that the irregular shape of the central contours may be correlated to different shapes of structural units exists in each region. Nonetheless, this may also cause by 
the different structural units for the respective region analyzed. The irregular shape of autocorrelation contour indicated that the $x$-axis is not representative plot of these functions.

\section{Summary}

Theoretically, the OR starts with the initial small crystalline nuclei formation that evolved into a larger particle size to some extent in a supersaturated reaction solution. The OR mechanism is considered as the primary path for crystal growth in chemical reaction systems. Since $\mathrm{ZnO}$ nanostructures prepared from the current work does not involve any reducing agent or growth agent, the OR is omitted.

Hence, OA is the primary control of the system nature. In the present work, the OA controls the assembly of the produced $\mathrm{ZnO}$ nanostructures through the inevitable repeating attachment events of merged nanostructures that usually occurred on specific latticematched crystal facets. The produced output of the current results is not adequate but offers one of the contributed conclusions on the proposed theory so far. The evidence for the corresponding $\mathrm{ZnO}$ nanostructures $\mathrm{OA}$ formation process becomes evident in which the nanostructure boundary is not an immediate process but instead depicts the assembly arrangement of broad range intermediate nanostructures between nanoparticles and to bulk crystal structures. The formation of intermediate nanostructures which is considered as a bridge for converting small crystals to aggregate crystals plays the primary role in the nanosurfaces changes.

The nanostructures obtained at different synthesis temperatures are considered as adjacent particles in which the tendency for the planes to create integrated structural trends (and not fusion) is higher. Hence, the surface structural changes undergone by the $\mathrm{ZnO}$ nanostructures formed at different reactant temperatures became the base reason for the inducement of the crystallization level. The interchange between kinetics (energy motion) and thermodynamics is the primary factor in determining the characteristics of $\mathrm{OA}$. The growth and coarsening of nanostructures started before the OA process. As the OA starts, the smooth surface condition of the nanostructures is achieved. The rough surface of the tiny small nanostructures is controlled by the crystal growth rate that is restrained by diffusion. Typically, such changes in the formation of the nanostructure give a significant effect in inducing suspension characteristics mainly when poorly dispersed nanostructures are formed.

Usually, the fusion structure gives better suspension characteristics of nanostructures due to the excellent stabilization in the liquid medium. However, the OA-grown $\mathrm{ZnO}$ nanostructures incline to cluster together, that is supposed to relate to the ability to reduce its overall surface energy by matching crystal lattices and thus decreases the exposed regions and defects. The types of growth processes during the nanostructure formation influence the nanostructure suspension surface.

However, as the TA is introduced into the samples, the acidic medium led to particle crystallite fusion. Based on the thermodynamic and dynamic mechanisms theories, the creation of stable crystalline phases in a solution should be led by the formation of metastable intermediate phases. Theoretically, the rough surface state of the nanostructures upon the TA introduction is caused due to the acidic medium that caused a lower nucleation energy barrier.

The direct autocorrelation using FFT embedded in a Gatan software indicated that the micrographs of the TEM showed the aberrations in the FFT autocorrelation associated with the presence of the aliasing effect. In the $x$-axis of the autocorrelation, the overlap units of the structure caused the $x$-lineout to dominantly appeared. The autocorrelation function needs to be studied further.

\section{Conclusions}

The present work demonstrated the effect of $\mathrm{pH}$ that commonly leads to the OA process in which the surface structure of adjacent $\mathrm{ZnO}$ nanostructures tends to attach and create the metastable structures before the size and morphological changes. The TA has 
a dominant role in the $\mathrm{pH}$-morphological and size-dependent characteristics of the $\mathrm{ZnO}$ nanostructures because of the rich phenolic hydrogen group of the ligand which caused the changes in the electron-donating system of the functional group on $\mathrm{ZnO}$ nanostructures. Once the alteration occurs, the hydrogen bonding between the $\mathrm{ZnO}$ nanostructures induced their agglomeration or dispersion. The correlation function performed shows the mimic condition of the TEM micrographs obtained in this work. It is hoped that the work could be one of the baselines for further exploration on the effect of surface functional group caused by the $\mathrm{pH}$ change and stimulates more theoretical and experiments studies related to the $\mathrm{ZnO}$ nanostructures.

Author Contributions: Conceptualisation, N.A.C.L. and S.T.; methodology, N.A.C.L.; software, N.A.C.L.; validation, N.A.C.L. and A.K.; formal analysis, N.A.C.L. and A.K.; investigation, N.A.C.L. and A.K.; resources, N.A.C.L.; data curation, N.A.C.L. and A.K.; writing-original draft preparation, N.A.C.L., S.T. and A.K.; writing-review and editing, N.A.C.L.; visualisation, N.A.C.L.; supervision, N.A.C.L.; project administration, N.A.C.L.; funding acquisition, N.A.C.L and S.T. All authors have read and agreed to the published version of the manuscript.

Funding: This research was funded by Universiti Malaysia Pahang (RDU190360, PGRS1903179 and PGRS200312), Oxford Martin School and EasternBiotech UK.

Acknowledgments: Universiti Malaysia Pahang and Universiti Malaya fully support the facilities and resources for this research. The main author (N.A.C.L.) would like to acknowledge the support of the internal grants of Universiti Malaysia Pahang (RDU190360, PGRS1903179 and PGRS200312). S.T. acknowledges support from DNANO. Ltd. UK.

Conflicts of Interest: We hereby confirm that the authors have no conflict of interest and the disclosure made for this manuscript is complete and correct to the best of our information and belief. We agree that if we become aware of any information that might indicate that this disclosure is inaccurate or that we have not complied with the conflict of interest policy, we will notify the journal immediately.

\section{References}

1. Penn, R.L.; Huisken, J.; Swoger, J.; Del Bene, F.; Wittbrodt, J.; Stelzer, E.H.K. Imperfect Oriented Attachment: Dislocation Generation in Defect-Free Nanocrystals. Science 1998, 281, 969-971. [CrossRef] [PubMed]

2. Penn, R.L.; Banfield, J.F. Oriented attachment and growth, twinning, polytypism, and formation of metastable phases; insights from nanocrystalline $\mathrm{TiO}_{2}$. Am. Mineral. 1998, 83, 1077-1082. [CrossRef]

3. Penn, R.L.; Soltis, J.A. Characterising crystal growth by oriented aggregation. CrystEngComm 2014, 16, 1409-1418. [CrossRef]

4. Padhi, S.K.; Krishna, M.G. Non-Classical Crystal Growth Recipe using nanocrystalline ceria a detailed review. arXiv 2019, arXiv:1911.07454.

5. Cao, D.; Gong, S.; Shu, X.; Zhu, D.; Liang, S. Preparation of ZnO Nanoparticles with High Dispersibility Based on Oriented Attachment (OA) Process. Nanoscale Res. Lett. 2019, 14, 1-11. [CrossRef]

6. Hazarika, S.; Mohanta, D. Oriented attachment (OA) mediated characteristic growth of $\mathrm{Gd}_{2} \mathrm{O}_{3}$ nanorods from nanoparticle seeds. J. Rare Earths 2016, 34, 158-165. [CrossRef]

7. Song, M.; Zhou, G.; Lu, N.; Lee, J.; Nakouzi, E.; Wang, H.; Li, D. Oriented attachment induces fivefold twins by forming and decomposing high-energy grain boundaries. Science 2019, 367, 40-45. [CrossRef]

8. Yang, J.; Zeng, Z.; Kang, J.; Betzler, S.; Czarnik, C.; Zhang, X.; Ophus, C.; Yu, C.; Bustillo, K.C.; Pan, M.; et al. Formation of two-dimensional transition metal oxide nanosheets with nanoparticles as intermediates. Nat. Mater. 2019, 18, 970-976. [CrossRef]

9. Sushko, M.L. Understanding the driving forces for crystal growth by oriented attachment through theory and simulations. $J$. Mater. Res. 2019, 34, 2914-2927. [CrossRef]

10. Lah, N.A.C.; Kamaruzaman, A. The optical quantification measurement on aggregated aqueous ZnO nanostructures upon exposure to tannic acid. Mater. Res. Express 2019, 6, 125046. [CrossRef]

11. Lah, N.A.C.; Mohamad, N.; Saari, M.M.; Johan, M.R. Nanoscopic tannic acid-ZnO colloid: Low temperature synthesis and the influence of $\mathrm{pH}$ on the aggregates. Mater. Res. Express 2019, 6, 065007. [CrossRef]

12. Rajan, A.K.; Cindrella, L. w-ZnO nanostructures with distinct morphologies: Properties and integration into dye sensitized solar cells. Ceram. Int. 2020, 46, 8174-8184. [CrossRef]

13. Basnet, P.; Chatterjee, S. Structure-directing property and growth mechanism induced by capping agents in nanostructured ZnO during hydrothermal synthesis-A systematic review. Nano Struct. Nano Objects 2020, 22, 100426. [CrossRef]

14. Kuo, C.T.; Lee, J.C.; Chen, Y.; Wu, Y.F. Structural Investigations on Hydrothermally Grown ZnO Nanostructures. In Key Engineering Materials; Trans Tech Publ.: Zürich, Switzerland, 2020. 
15. Thbayh, D.K.; Talib, R.A.; Ziadan, K.M. The effect of polyaniline addition on ZnO for the fabrication of high performance photodetection. MSEE 2020, 757, 012074. [CrossRef]

16. Nanev, C.N. Advancements (and challenges) in the study of protein crystal nucleation and growth; thermodynamic and kinetic explanations and comparison with small-molecule crystallisation. Prog. Cryst. Growth Charact. Mater. 2020, 66, 100484. [CrossRef]

17. Cozzoli, P.D.; Nobile, C. Colloidal oxide-based heterostructured nanocrystals. In Colloidal Metal Oxide Nanoparticles; Elsevier: Amsterdam, The Netherlands, 2020; pp. 401-470.

18. Gerbreders, V.; Krasovska, M.; Sledevskis, E.; Gerbreders, A.; Mihailova, I.; Tamanis, E.; Ogurcovs, A. Hydrothermal synthesis of $\mathrm{ZnO}$ nanostructures with controllable morphology change. CrystEngComm 2020, 22, 1346-1358. [CrossRef]

19. Laskar, I.B.; Rokhum, S.L.; Gupta, R.; Chatterjee, S. Zinc oxide supported silver nanoparticles as a heterogeneous catalyst for production of biodiesel from palm oil. Environ. Prog. Sustain. Energy 2020, 39, 13369. [CrossRef]

20. Vill, R.; Gülcher, J.; Khalatur, P.; Wintergerst, P.; Stoll, A.; Mourran, A.; Ziener, U. Supramolecular polymerisation: Challenges and advantages of various methods in assessing the aggregation mechanism. Nanoscale 2019, 11, 663-674. [CrossRef]

21. Liu, C.; Cheng, Y.-B.; Ge, Z. Understanding of perovskite crystal growth and film formation in scalable deposition processes. Chem. Soc. Rev. 2020, 49, 1653-1687. [CrossRef]

22. Jose, N.; Lapkin, A. Influence of Hydrodynamics on Wet Syntheses of Nanomaterials. In Advanced Nanomaterials for Catalysis and Energy; Elsevier: Amsterdam, The Netherlands, 2019; pp. 29-59.

23. Rauf, A.; Abbas, Z.; Shehzad, S.A. Interactions of Active and Passive Control of Nanoparticles on Radiative Magnetohydrodynamics Flow of Nanofluid Over Oscillatory Rotating Disk in Porous Medium. J. Nanofluids 2019, 8, 1385-1396. [CrossRef]

24. Sharaf, O.Z.; Al-Khateeb, A.N.; Kyritsis, D.C.; Abu-Nada, E. Four-way coupling of particle-wall and colloidal particle-particle interactions in direct absorption solar collectors. Energy Convers. Manag. 2019, 195, 7-20. [CrossRef]

25. Loza, K.; Epple, M.; Maskos, M. Stability of Nanoparticle Dispersions and Particle Agglomeration. In Biological Responses to Nanoscale Particles; Springer: Berlin/Heidelberg, Germany, 2019; pp. 85-100.

26. Zareei, M.; Yoozbashizadeh, H.; Hosseini, H.R.M. Investigating the effects of $\mathrm{pH}$, surfactant and ionic strength on the stability of alumina/water nanofluids using DLVO theory. J. Therm. Anal. Calorim. 2018, 135, 1185-1196. [CrossRef]

27. Lu, K.; Gervasio, M. Simulation Study of Nanoparticle-Polymer Organic Suspension Stability. Adv. Theory Simul. 2019, 2, 1900010. [CrossRef]

28. Chaturbedi, A.K. A Combined Computational and Experimental Study of the Heteroaggregation of Dissimilar Adsorbent Particles; Rutgers University-School of Graduate Studies, Rutgers University: New Brunswick, NJ, USA, 2019.

29. Shrestha, S.; Wang, B.; Dutta, P. Nanoparticle processing: Understanding and controlling aggregation. Adv. Colloid Interface Sci. 2020, 279, 102162. [CrossRef] [PubMed]

30. Wade, J.B. Physicochemical Principles Governing Agglomeration and Growth Kinetics. In Handbook of Pharmaceutical Wet Granulation; Elsevier: Amsterdam, The Netherlands, 2019; pp. 3-35.

31. Savoie, W.C. Effect of Shape and Particle Coordination on Collective Dynamics of Granular Matter; Georgia Institute of Technology: Atlanta, GA, USA, 2019.

32. Nau, M. Functional Surface Coatings and Novel Materials Derived from Hydroxypropyl Cellulose; Technische Universität Berlin: Berlin, Germany, 2020.

33. Rakhsha, A.H.; Abdizadeh, H.; Pourshaban, E.; Golobostanfard, M.R.; Mastelaro, V.R.; Montazerian, M. Ag and Cu doped ZnO nanowires: A pH-Controlled synthesis via chemical bath deposition. Materialia 2019, 5, 100212. [CrossRef]

34. Sun, X.; Liu, C.; Omer, A.M.; Lu, W.; Zhang, S.; Jiang, X.; Wu, H.; Yu, D.; Ouyang, X.-K. pH-sensitive ZnO/carboxymethyl cellulose/chitosan bio-nanocomposite beads for colon-specific release of 5-fluorouracil. Int. J. Biol. Macromol. 2019, 128, 468-479. [CrossRef]

35. Bian, S.-W.; Mudunkotuwa, I.A.; Rupasinghe, T.; Grassian, V.H. Aggregation and Dissolution of 4 nm ZnO Nanoparticles in Aqueous Environments: Influence of $\mathrm{pH}$, Ionic Strength, Size, and Adsorption of Humic Acid. Langmuir 2011, 27, 6059-6068. [CrossRef]

36. Jang, J.-M.; Kim, S.-D.; Choi, H.-M.; Kim, J.-Y.; Jung, W.-G. Morphology change of self-assembled ZnO 3D nanostructures with different $\mathrm{pH}$ in the simple hydrothermal process. Mater. Chem. Phys. 2009, 113, 389-394. [CrossRef]

37. Esmaeili-Zare, M.; Behpour, M. CIS/CdS/ZnO/ZnO:Al modified photocathode for enhanced photoelectrochemical behavior under visible irradiation: Effects of $\mathrm{pH}$ and concentration of electrolyte solution. Int. J. Hydrogen Energy 2020, 45, 8273-8281. [CrossRef] 